\title{
Use of coronary calcium score scans from stand-alone multislice computed tomography for attenuation correction of myocardial perfusion SPECT
}

Schepis, T ; Gaemperli, O ; Koepfli, P ; Rüegg, C ; Burger, C ; Leschka, S ; Desbiolles, L ; Husmann, L ; Alkadhi, H ; Kaufmann, P A

\begin{abstract}
PURPOSE: To evaluate the use of CT attenuation maps, generated from coronary calcium scoring (CCS) scans at in- and expiration with a 64-slice CT scanner, for attenuation correction (AC) of myocardial perfusion SPECT images. METHODS: Thirty-two consecutive patients underwent( $99 \mathrm{~m}) \mathrm{Tc}-$ tetrofosmin gated adenosine stress/rest SPECT scan on an Infinia Hawkeye SPECT-CT device (GE Medical Systems) followed by CCS and CT angiography on a 64-slice CT. AC of the iteratively reconstructed images was performed with AC maps obtained: (a) from the "Hawkeye" low-resolution X-ray CT facility attached to the Infinia camera (IRAC); (b) from the CCS scan acquired on a 64-slice CT scanner during maximal inspiration $(\mathrm{AC}(\mathrm{INSP}))$ and $(\mathrm{c})$ during normal expiration $(\mathrm{AC}(\mathrm{EXP}))$. Automatically determined uptake values of stress scans (QPS, Cedars Medical Sinai) from AC(INSP) and AC(EXP) were compared with IRAC. Agatston score (AS) values using AC(INSP)versus AC(EXP) were also compared. RESULTS: AC(INSP) and AC(EXP) resulted in identical findings versus IRAC by visual analysis. A good correlation for uptake values between IRAC and $\mathrm{AC}(\mathrm{INSP}$ ) was found (apex, $\mathrm{r}=0.92$; anterior, $\mathrm{r}=0.85$; septal, $\mathrm{r}=0.91$; lateral, $\mathrm{r}=0.86$; inferior, $\mathrm{r}=0.90$; all $\mathrm{p}<0.0001)$. The correlation was even closer between IRAC and $\mathrm{AC}(\mathrm{EXP})$ (apex, $\mathrm{r}=0.97$; anterior, $\mathrm{r}=0.91$; septal, $\mathrm{r}=0.94$; lateral, $\mathrm{r}=0.92$; inferior, $\mathrm{r}=0.97$; all $\mathrm{p}<0.0001)$. The mean AS during inspiration $(319+/-737)$ and expiration $(317+/-778)$ was comparable $(\mathrm{p}=\mathrm{NS})$. CONCLUSION: Attenuation maps from CCS allow accurate AC of SPECT MPI images. $\mathrm{AC}(\mathrm{EXP})$ proved superior to $\mathrm{AC}(\mathrm{INSP})$, suggesting that in hybrid scans CCS may be performed during normal expiration to allow its additional use for AC of SPECT MPI.
\end{abstract}

DOI: https://doi.org/10.1007/s00259-006-0173-8

Posted at the Zurich Open Repository and Archive, University of Zurich ZORA URL: https://doi.org/10.5167/uzh-13987

Journal Article

Published Version

Originally published at:

Schepis, T; Gaemperli, O; Koepfli, P; Rüegg, C; Burger, C; Leschka, S; Desbiolles, L; Husmann, L; Alkadhi, H; Kaufmann, P A (2007). Use of coronary calcium score scans from stand-alone multislice computed tomography for attenuation correction of myocardial perfusion SPECT. European Journal of Nuclear Medicine and Molecular Imaging, 34(1):11-19.

DOI: https://doi.org/10.1007/s00259-006-0173-8 


\title{
Use of coronary calcium score scans from stand-alone multislice computed tomography for attenuation correction of myocardial perfusion SPECT
}

\author{
Tiziano Schepis ${ }^{1,4}$, Oliver Gaemperli1, 4, Pascal Koepfli ${ }^{11}{ }^{4}$, Christine Rüegg ${ }^{1}$, Cyrill Burger ${ }^{1}$, Sebastian Leschka ${ }^{2}$, \\ Lotus Desbiolles², Lars Husmann², Hatem Alkadhi², Philipp A. Kaufmann1, 3, 4 \\ ${ }^{1}$ Clinic of Nuclear Medicine, Nuclear Cardiology, University Hospital Zurich, Zurich, Switzerland \\ 2 Institute of Diagnostic Radiology, University Hospital Zurich, Zurich, Switzerland \\ ${ }^{3}$ Centre for Integrative Human Physiology, University of Zurich, Zurich, Switzerland \\ ${ }^{4}$ Cardiovascular Center, University Hospital Zurich, Raemistrasse 100, Zurich, Switzerland
}

Received: 10 March 2006 / Accepted: 11 May 2006 / Published online: 4 August 2006

(C) Springer-Verlag 2006

\begin{abstract}
Purpose: To evaluate the use of CT attenuation maps, generated from coronary calcium scoring (CCS) scans at in- and expiration with a 64-slice CT scanner, for attenuation correction (AC) of myocardial perfusion SPECT images.

Methods: Thirty-two consecutive patients underwent ${ }^{99 \mathrm{~m}}$ Tc-tetrofosmin gated adenosine stress/rest SPECT scan on an Infinia Hawkeye SPECT-CT device (GE Medical Systems) followed by CCS and CT angiography on a 64-slice CT. AC of the iteratively reconstructed images was performed with AC maps obtained: (a) from the "Hawkeye" low-resolution X-ray CT facility attached to the Infinia camera (IRAC); (b) from the CCS scan acquired on a 64slice $\mathrm{CT}$ scanner during maximal inspiration $\left(\mathrm{AC}_{\mathrm{INSP}}\right)$ and (c) during normal expiration $\left(\mathrm{AC}_{\mathrm{EXP}}\right)$. Automatically determined uptake values of stress scans (QPS, Cedars Medical Sinai) from $\mathrm{AC}_{\mathrm{INSP}}$ and $\mathrm{AC}_{\mathrm{EXP}}$ were compared with IRAC. Agatston score (AS) values using $\mathrm{AC}_{\mathrm{INSP}^{-}}$ versus $\mathrm{AC}_{\mathrm{EXP}}$ were also compared.

Results: $\mathrm{AC}_{\mathrm{INSP}}$ and $\mathrm{AC}_{\mathrm{EXP}}$ resulted in identical findings versus IRAC by visual analysis. A good correlation for uptake values between IRAC and $\mathrm{AC}_{\mathrm{INSP}}$ was found (apex, $r=0.92$; anterior, $r=0.85$; septal, $r=0.91$; lateral, $r=0.86$; inferior, $r=0.90$; all $p<0.0001)$. The correlation was even closer between IRAC and $\mathrm{AC}_{\mathrm{EXP}}$ (apex, $r=0.97$; anterior, $r=0.91$; septal, $r=0.94$; lateral, $r=0.92$; inferior, $r=0.97$; all $p<0.0001)$. The mean AS during inspiration (319 \pm 737$)$ and expiration $(317 \pm 778)$ was comparable $(p=\mathrm{NS})$.
\end{abstract}

Philipp A. Kaufmann (

Cardiovascular Center,

University Hospital Zurich,

Raemistrasse 100,

8091 Zurich, Switzerland

e-mail: pak@usz.ch

Tel.: +41-1-2553555, Fax: +41-1-2554414
Conclusion: Attenuation maps from CCS allow accurate $\mathrm{AC}$ of SPECT MPI images. $\mathrm{AC}_{\mathrm{EXP}}$ proved superior to $\mathrm{AC}_{\mathrm{INSP}}$, suggesting that in hybrid scans $\mathrm{CCS}$ may be performed during normal expiration to allow its additional use for AC of SPECT MPI.

Keywords: SPECT-CT - Attenuation correction -

Multislice computed tomography -

Coronary calcium score

Eur J Nucl Med Mol Imaging (2007) 34:11-19

DOI 10.1007/s00259-006-0173-8

\section{Introduction}

Non-uniform soft tissue photon attenuation adversely affects the diagnostic accuracy of single-photon emission computed tomography (SPECT) myocardial perfusion imaging (MPI) [1]. Several methods for attenuation correction have been proposed [2], most of them using attenuation maps based on radionuclide line sources [3-5]. Recently, however, the use of computed tomography (CT) attenuation correction (AC) has been introduced [6] and established for MPI SPECT [7 , 8], as well as for quantitative positron emission tomography MPI $[1,9]$. Since CT can acquire images with a far higher spatial resolution and higher photon flux, the transmission scan can be acquired in a much shorter time and with higher quality than can be obtained from a conventional radionuclide transmission scan. CT has been shown to yield good results for AC [10], especially if correction for misalignment between SPECT and the attenuation map is performed [8]. Considering the widespread use of SPECT MPI and the increasing interest in multislice $\mathrm{CT}$ for the non-invasive assessment of patients with known or suspected coronary artery disease (CAD), 
it appears important in view of the upcoming generation of hybrid SPECT-CT scanners to evaluate whether multislice CT data from coronary calcium scoring (CCS) can also be used for generation of reliable attenuation maps to correct MPI SPECT. It has been previously shown that the respiration-dependent change in attenuation values is a possible drawback of CT-based AC $[11,12]$. The attenuation map obtained with the lower resolution X-ray-based CT data (obtained with a matrix of $128 \times 128$ ) of hybrid SPECTCT systems represents an average over many breathing cycles. By contrast, data acquisition with a modern multislice CT scanner occurs within fractions of a breath-hold [1].

The purpose of this study was, therefore, to prospectively compare the effect of AC on iteratively reconstructed SPECT images with the "Hawkeye" low-resolution X-ray CT facility attached to the Infinia camera (IRAC) versus high-resolution CT attenuation maps generated from CCS with a 64-slice CT scanner during inspiration or expiration.

\section{Materials and methods}

\section{Study population}

We prospectively analysed 32 patients (15 females, 17 males) referred for gated SPECT for known or suspected CAD. Their mean age was $66 \pm 11$ years (range $41-89$ years), and their mean body mass index, $29 \pm 4 \mathrm{~kg} / \mathrm{m}^{2}$. Six patients had a history of previous myocardial infarction (two anterior wall infarctions and four inferior wall infarctions). Each patient subsequently underwent CCS and CT angiography on a 64-slice CT scanner. The mean time interval between SPECT and CT angiography was $1 \pm 4$ days. The study protocol was approved by the local ethics committee and written informed consent was obtained from all subjects.

\section{SPECT imaging}

All patients underwent a 1-day stress $(0.14 \mathrm{mg} / \mathrm{kg} / \mathrm{min}$ adenosine i.v. $) /$ rest MPI protocol using a dose of $300 \mathrm{MBq}$ and $900 \mathrm{MBq}$ of ${ }^{99 \mathrm{~m}} \mathrm{Tc}-$ tetrofosmin, respectively. Data acquisition was performed on a hybrid SPECT-CT dual-head detector camera with the Hawkeye facility (Infinia, General Electric Medical Systems, Milwaukee, WI, USA). Emission data were acquired with a parallel-hole, low-energy, highresolution collimator with a $20 \%$ symmetric window centered at $140 \mathrm{keV}$. Further acquisition parameters were $3^{\circ}$ rotation per stop, $180^{\circ}$ each head, and $25 \mathrm{~s}$ per projection. Acquisitions were gated for 16 frames per R-R cycle with an acceptance window of 50\%. Immediately after acquisition of SPECT images, a low-dose CT for AC was performed by use of the GE Hawkeye system [6] with the following parameters: $140 \mathrm{kV}, 3.0 \mathrm{~mA}$, with a single slice being imaged in about $14 \mathrm{~s}$. CT images were reconstructed at a $10-\mathrm{mm}$ section thickness by using a standard reconstruction algorithm with a $128 \times 128$ matrix and a full-chest-size-adapted field of view $(\mathrm{FOV})$ of $50 \mathrm{~cm} \times 50 \mathrm{~cm}$.

\section{CT imaging}

For CCS each patient underwent a 64-slice CT examination on a LightSpeed VCT Scanner (General Electric Medical Systems, Milwaukee, WI, USA). Previous studies have suggested that acquisition of the $\mathrm{CT}$ attenuation map with free breathing and postexhalation breath-hold resulted in better matching with SPECT and PET images than did acquisition with post-inhalation breath-hold $[11,12]$. By contrast, CCS is routinely performed during deep inspiration. Therefore, two CT scans for attenuation correction-one during maximum inspiration and one during normal expirationwere performed, covering the entire heart using prospectively ECGtriggered sequential images at $70 \%$ of the R-R interval, $2.5-\mathrm{mm}$ section thickness, and $0.35 \mathrm{~s}$ gantry rotation time at $120 \mathrm{kV}$ and 200 $250 \mathrm{~mA}$, depending on the patient's size. Normal expiration was defined as the respiration level achieved when a patient, after inspiration and expiration, held his or her breath without forcefully exhaling. CT images were reconstructed at a $5.0-\mathrm{mm}$ section thickness by using a reconstruction algorithm with a $512 \times 512$ matrix and a full-chest-size-adapted FOV of $50 \mathrm{~cm} \times 50 \mathrm{~cm}$. The reconstructed CT images were then transferred to the Xeleris workstation (GE Medical Systems, Milwaukee, WI, USA).

\section{CT attenuation correction of emission images}

All stress and rest SPECT images were iteratively reconstructed using three different types of CT-based transmission data: (a) using the "Hawkeye" low-resolution X-ray CT data (IRAC) as a standard, (b) using the CCS scan data generated with the 64-slice CT obtained during deep inspiration $\left(\mathrm{AC}_{\mathrm{INSP}}\right)$ and $(\mathrm{c})$ during normal expiration $\left(\mathrm{AC}_{\mathrm{EXP}}\right)$. For processing purposes the CT and SPECT data were loaded into a prototype version of the enhanced InfiniaQGS protocol within the Xeleris 1.1427 software. In a first step the CT images were fused with the uncorrected SPECT images to verify the alignment, as previously reported [8]. When necessary, the location of the CT images was interactively adjusted by shifting and/or rotating the images in all directions until the myocardium visible in the SPECT images was entirely overlying heart tissue in the CT images, following as closely as possible the CT myocardium contours (Fig. 1). The aligned CT images were then transformed into SPECT attenuation maps by the following steps: First, the CT images were reformatted into a volume with the same voxel and matrix size as the SPECT images. Then, the CT Hounsfield values were transformed into linear attenuation coefficients corresponding to the energy of the SPECT emission photons. For CT values below 0, attenuation was assumed to have an energy dependence similar to water, while CT values above 0 were treated as a mixture of bone and water. Hereby, the transformation considered the effective CT energy spectrum. Next, the attenuation maps were smoothed in all three directions with a Gaussian filter to adjust the resolution to that of the SPECT data, taking into account the SPECT voxel size. Finally, the CT-based attenuation coefficients were included in an iterative ordered subsets emission maximisation algorithm (OSEM, two iterations, ten subsets, Butterworth filter with critical frequency 0.25 and power $5.0)$ to reconstruct attenuation-corrected SPECT images. The resulting transaxial slice images had a $64 \times 64$ matrix and voxel dimensions of $6.8 \times 6.8 \times 6.8 \mathrm{~mm}^{3}$.

\section{Visual analysis of attenuation-corrected SPECT images}

Consensus reading with regard to the presence and location of reversible and/or fixed perfusion defects in five left ventricular regions (apical, anterior, septal, lateral, and inferior) was performed by two experienced nuclear cardiologists on short-axis, horizontal and vertical long-axis slices as well as the polar maps. The results from $\mathrm{AC}_{\mathrm{INSP}}$ and $\mathrm{AC}_{\mathrm{EXP}}$ were compared with those obtained from IRAC. In cases of disagreement between the three AC methods, CT 
coronary angiography, which was obtained after CCS in all patients, was available as an external reference.

Quantitative regional uptake values, defect extent and summed perfusion scores

Attenuation-corrected stress and rest SPECT images obtained using either IRAC, $\mathrm{AC}_{\mathrm{INSP}}$ or $\mathrm{AC}_{\mathrm{EXP}}$ were displayed as short-axis, horizontal and vertical long-axis slices using the QPS/QGS software package (Cedars-Sinai Medical Center, Los Angeles, CA, USA) [13]. Perfusion SPECT images were analysed using a 20 -segment model for the left ventricle [14]. Polar maps were normalised to $100 \%$ peak activity and relative percentage counts uptake of gamma ray emissions was assessed and automatically rated for each of the 20 myocardial segments using a five-point scale $(0=$ normal uptake, $1=$ mildly reduced uptake, $2=$ moderately reduced uptake, $4=$ severely reduced uptake and $4=$ no uptake), as previously described $[15,16]$. Briefly, the summed stress score (SSS) and summed rest score (SRS) were calculated as the sums of scores of the 20 segments in the stress and rest images, respectively. The percent uptake values are only provided for stress scans, as these were performed with a low dose activity according to the protocol and, therefore, were potentially more prone to artefacts introduced by AC. In addition, the quantitative defect extent was expressed as a percentage of the total left ventricular myocardium. Because the effect of AC may vary substantially from region to region owing to anatomical nonuniformity, as demonstrated by Ficaro et al. [17], we assigned the 20 segments of the left ventricular myocardium to five regions of the left ventricle: apex (segments 19 and 20), anterior (segments 1, 2, 7, 8, 13 and 14), septal (segments 3, 9 and 15), lateral (segments 5, 6, 11, 12, 17 and 18), and inferior (segments 4, 10 and 16).

\section{Coronary calcium scoring}

CCS was quantified for the two CT scans obtained by $\mathrm{AC}_{\mathrm{INSP}}$ and $\mathrm{AC}_{\mathrm{EXP}}$. CT images were reconstructed at a $3.0-\mathrm{mm}$ section thickness by using a reconstruction algorithm with a $512 \times 512$ matrix and a FOV of $25 \mathrm{~cm} \times 25 \mathrm{~cm}$. Coronary artery lesions were manually planimetered using the SmartScore software on a GE Advantage Windows workstation version 4.2 (GE Medical Systems, Milwaukee, WI, USA) to obtain the total calcium burden in the coronary arteries, providing the Agatston Score Equivalent for a multi-detector CT

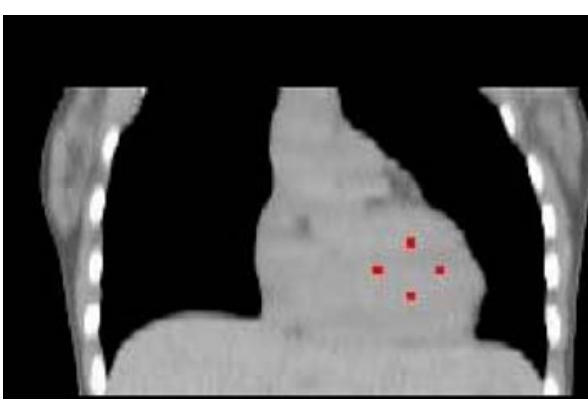

CT Coronal

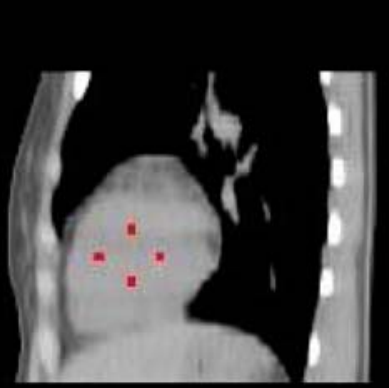

CT Sagittal

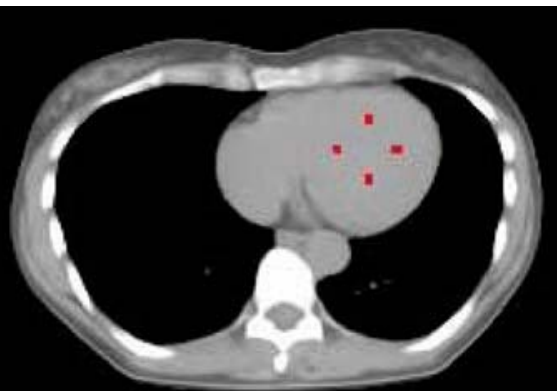

CT Transaxial:
NM Coronal

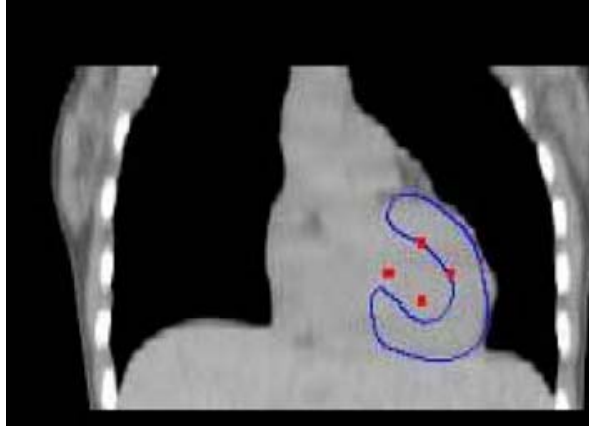

Fused Coronal
NM Sagittal

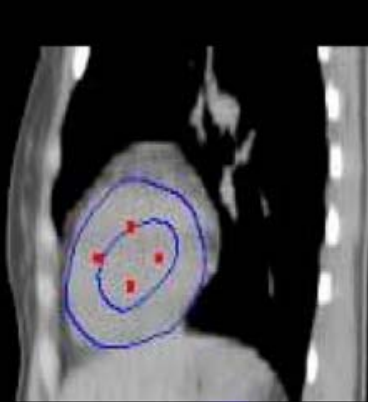

Fused Sagittal
NM Transaxial

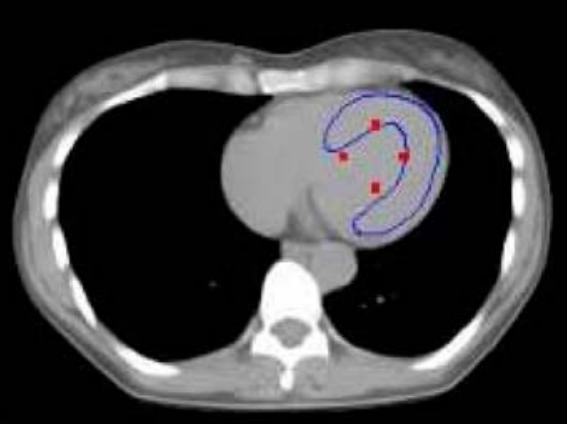

Fused Transaxial

Fig. 1. Coronal, sagittal and transaxial CT images were fused with the non-corrected SPECT images to verify the alignment of the coregistration. When necessary, misalignment was corrected by manually adjusting CT images to best match MPI SPECT images 
Table 1. Mean values of defect extent and summed perfusion scores

\begin{tabular}{lccc}
\hline & IRAC & $\mathrm{AC}_{\mathrm{INSP}}$ & $\mathrm{AC}_{\mathrm{EXP}}$ \\
\hline All patients $(n=32)$ & & & \\
Stress defect extent (\%) & $5.9 \pm 7.5$ & $5.5 \pm 5.7$ & $5.7 \pm 7.2$ \\
Rest defect extent $(\%)$ & $3.6 \pm 6.4$ & $3.4 \pm 6.6$ & $3.5 \pm 6.2$ \\
SSS & $4.4 \pm 5.6$ & $3.9 \pm 5.3$ & $4.2 \pm 6.2$ \\
SDS & $2.5 \pm 3.0$ & $1.8 \pm 2.2$ & $2.1 \pm 2.4$ \\
SRS & $1.9 \pm 4.0$ & $2.3 \pm 4.4$ & $2.1 \pm 4.9$ \\
Patients with stress defects $(n=10)$ & & & \\
Stress defect extent $(\%)$ & $12.8 \pm 9.5$ & $13.3 \pm 9.5$ & $12.3 \pm 8.9$ \\
SSS & $9.3 \pm 6.9$ & $9.0 \pm 6.5$ & $9.0 \pm 8.8$ \\
Patients with rest defects $(n=6)$ & & & \\
Rest defect extent $(\%)$ & $11.2 \pm 11.9$ & $11.4 \pm 12.3$ & $11.0 \pm 11.6$ \\
SRS & $7.7 \pm 6.4$ & $8.7 \pm 6.6$ & $8.7 \pm 8.8$ \\
\hline
\end{tabular}

All comparisons between AC methods revealed $p=\mathrm{NS}$

IRAC attenuation correction (AC) using the Hawkeye low-resolution X-ray CT data, $A C_{I N S P} \mathrm{AC}$ using the CCS scan data obtained during inspiration, $A C_{E X P} \mathrm{AC}$ using the CCS scan data obtained during expiration, $S S S$ summed stress score, $S D S$ summed difference score, $S R S$ summed rest score

acquisition [18], where coronary calcification was defined as a lesion with an area greater than $1 \mathrm{~mm} \times 1 \mathrm{~mm}$ and a peak intensity greater than 130 Hounsfield units. CCS was determined for the four main coronary arteries in all slices and summed to generate the total score.

\section{Statistical analysis}

Values are expressed as mean \pm standard deviation. Pearson correlation coefficient and Bland and Altman analysis [19] were used for continuous values, while a chi-squared test was used to compare categorical visual analysis of $\mathrm{AC}_{\mathrm{INSP}}$ and $\mathrm{AC}_{\mathrm{EXP}}$ against IRAC. To assess the reliability for the retrospective alignment between the uncorrected SPECT and CT images, intra- and inter-observer reproducibility for quantitative regional uptake values using $\mathrm{AC}_{\mathrm{INSP}}$ and $\mathrm{AC}_{\mathrm{EXP}}$ was determined for the first ten consecutive patients using linear regression analysis. Differences in mean values for summed perfusion scores and defect extent between IRAC, $\mathrm{AC}_{\mathrm{INSP}}$ and $\mathrm{AC}_{\mathrm{EXP}}$ were compared by using analysis of variance for repeated measures (ANOVA). Differences in mean values for coronary calcium score between $\mathrm{AC}_{\mathrm{INSP}}$ and $\mathrm{AC}_{\mathrm{EXP}}$ were compared using a two-sided paired Student's $t$ test. For all analyses, $p<0.05$ indicated a statistically significant difference. All statistical analyses were performed using a commercially available software package (StatView for Windows, Version 5.0.1, SAS Institute, Cary, NC, USA).

\section{Results}

\section{Visual analysis of attenuation-corrected SPECT images}

Visual analysis with IRAC revealed fixed perfusion defects in six patients (two antero-apical infarctions, four inferior infarctions) and reversible defects in four patients (apical, anterior, lateral, and inferior ischaemia, $n=1$ each). In six of the remaining 22 patients there were minimal defects attributable to edge phenomena and fringe effects of the septum, which were, therefore, not considered to be perfusion defects. $\mathrm{AC}_{\mathrm{INSP}}$ and $\mathrm{AC}_{\mathrm{EXP}}$ showed identical findings compared with IRAC by visual analysis $(p<0.0001$ for chi-squared test).

\section{Defect extent and summed perfusion scores}

The mean values of rest and stress defects as well as the summed scores for IRAC, $\mathrm{AC}_{\mathrm{INSP}}$ and $\mathrm{AC}_{\mathrm{EXP}}$ are given in Table 1 . No statistically significant difference regarding the defect extent and the summed perfusion scores was found between either AC method ( $p=\mathrm{NS}$ for ANOVA). Excellent correlations were found for the SSS between IRAC and $\mathrm{AC}_{\text {INSP }}(r=0.88, \quad p<0.0001)$ and between IRAC and $\operatorname{AC}_{\mathrm{EXP}}(r=0.95, p<0.0001)$. Similarly, excellent correlations were obtained for the SRS between IRAC and $\operatorname{AC}_{\mathrm{INSP}}(r=0.95, \quad p<0.0001)$ and between IRAC and $\operatorname{AC}_{\mathrm{EXP}}(r=0.97, p<0.0001)$, while the correlation between IRAC and $\mathrm{AC}_{\mathrm{INSP}}$ and between IRAC and $\mathrm{AC}_{\mathrm{EXP}}$ for the

Table 2. Localisation and degree of MPI defects

\begin{tabular}{|c|c|c|c|c|c|c|}
\hline \multirow[t]{2}{*}{ Patient no. } & \multirow[t]{2}{*}{ Sex/age (yrs) } & \multirow[t]{2}{*}{ Localisation of MPI defect } & \multirow[t]{2}{*}{ Diseased artery } & \multicolumn{3}{|c|}{ Stress defect extent $(\%)$} \\
\hline & & & & IRAC & $\mathrm{AC}_{\mathrm{INSP}}$ & $\mathrm{AC}_{\mathrm{EXP}}$ \\
\hline 1 & $\mathrm{M} / 75$ & Antero-apical scar & LAD & 33 & 34 & 32 \\
\hline 10 & $\mathrm{M} / 68$ & Inferior scar & RCA & 23 & 22 & 21 \\
\hline 11 & $\mathrm{~F} / 68$ & Lateral ischaemia & LCX & 6 & 8 & 6 \\
\hline 12 & $\mathrm{~F} / 56$ & Apical ischaemia & LAD & 3 & 3 & 3 \\
\hline 13 & $\mathrm{~F} / 78$ & Anterior ischaemia & LAD & 10 & 11 & 9 \\
\hline 21 & $\mathrm{M} / 89$ & Inferior scar & RCA & 10 & 12 & 10 \\
\hline 26 & $\mathrm{M} / 71$ & Inferior ischaemia & RCA & 15 & 16 & 15 \\
\hline 30 & $\mathrm{M} / 69$ & Inferior scar & RCA & 17 & 15 & 16 \\
\hline 31 & $\mathrm{~F} / 77$ & Antero-apical scar & LAD & 9 & 11 & 9 \\
\hline 32 & $\mathrm{M} / 88$ & Inferior scar & $\mathrm{RCA}$ & 3 & 3 & 3 \\
\hline
\end{tabular}

$L A D$ left anterior descending coronary artery, $L C X$ left circumflex coronary artery, $R C A$ right coronary artery, $M P I$ myocardial perfusion imaging 
Fig. 2. Linear regression analysis and Bland-Altman plots for percent radiotracer uptake between IRAC and $\mathrm{AC}_{\mathrm{INSP}}$ and between IRAC and $\mathrm{AC}_{\mathrm{EXP}}$ in the apical (a), anterior (b), septal (c), lateral (d) and inferior (e) myocardial regions a

Apex

IRAC vS AC INSP
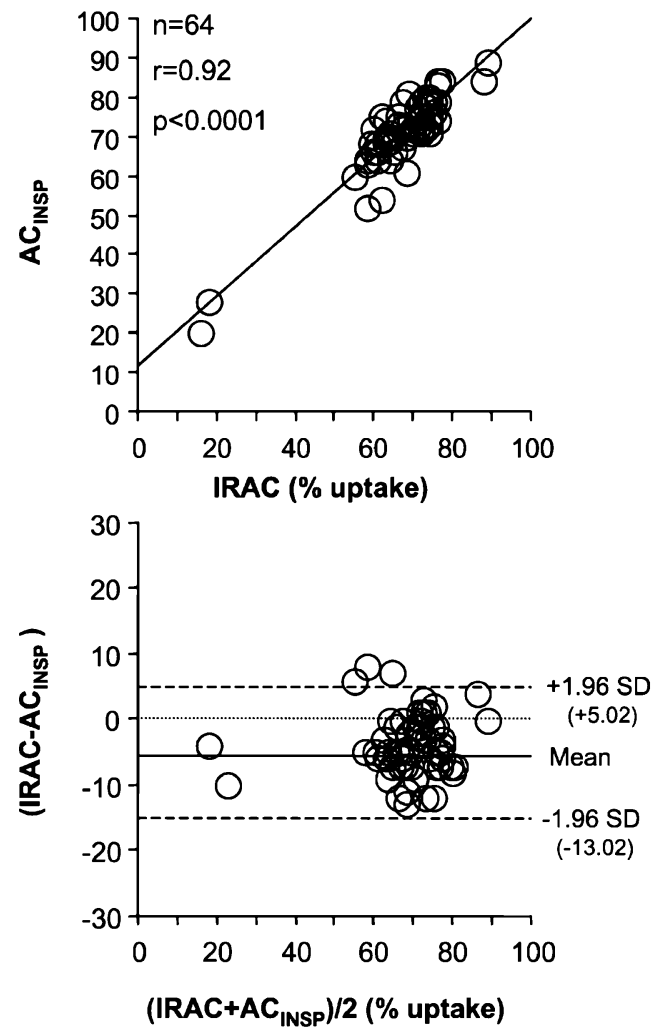

b

Anterior IRAC vs AC INSP $_{\text {}}$
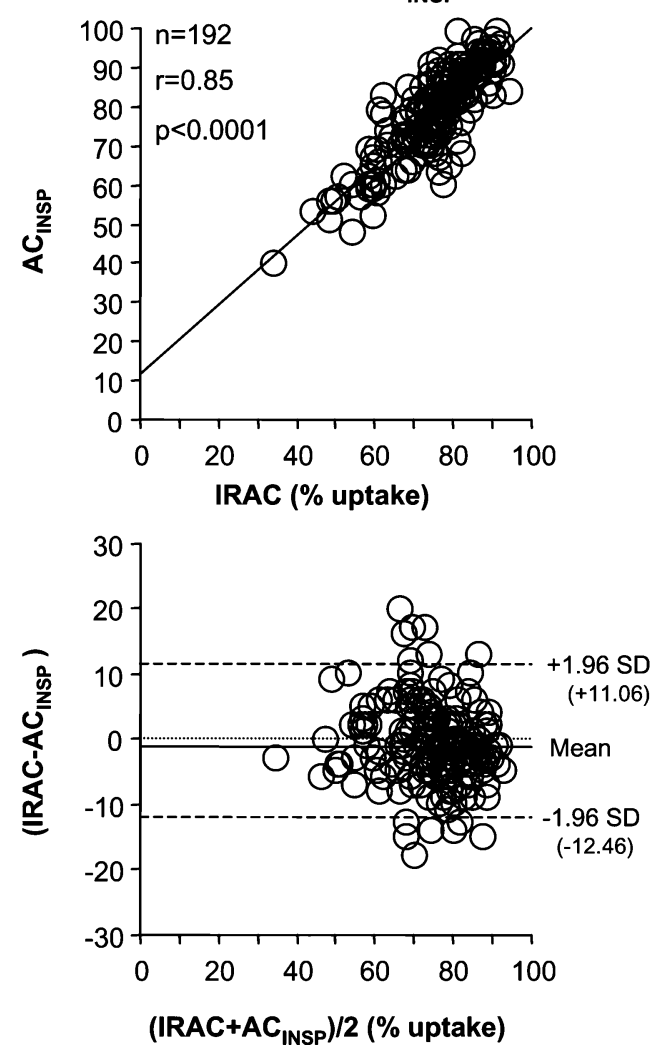

Apex

IRAC vS AC EXP $_{\text {}}$
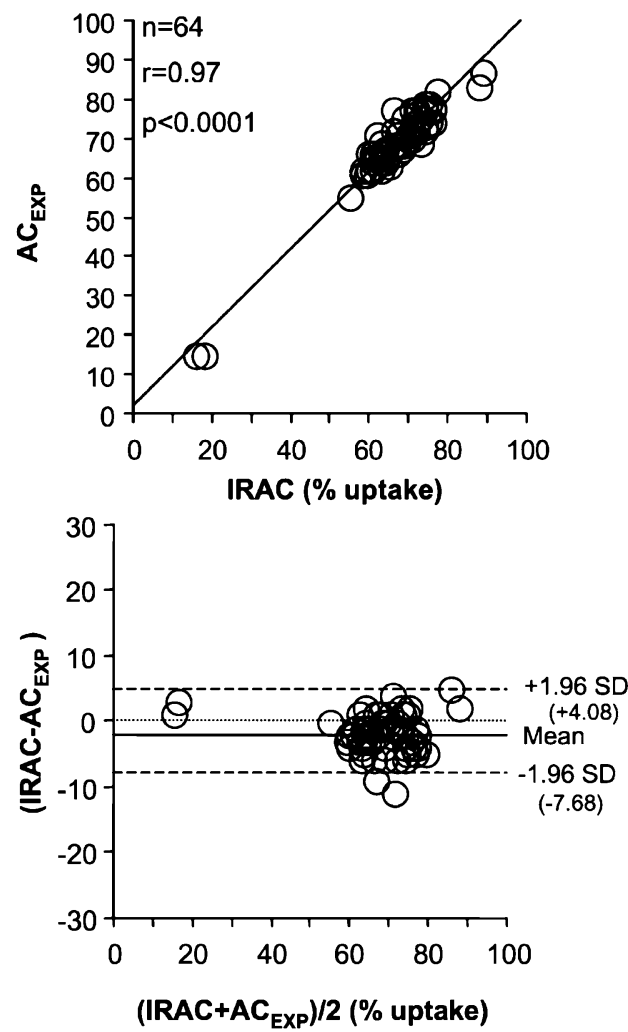

Anterior

IRAC vS AC
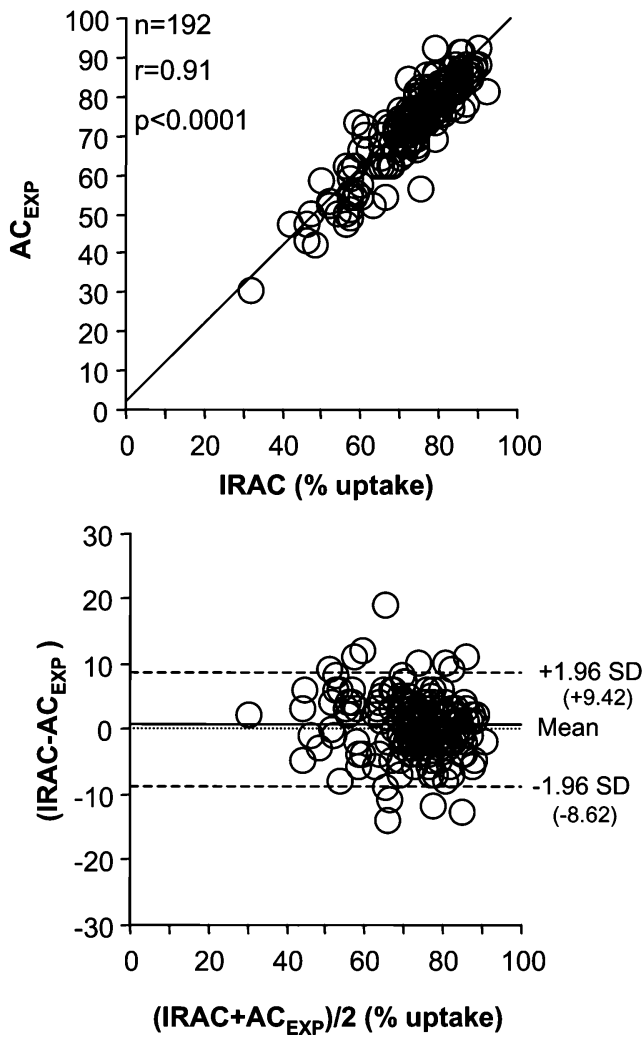
Fig. 2 (continued) c

Septum

IRAC vS AC $_{\text {INSP }}$
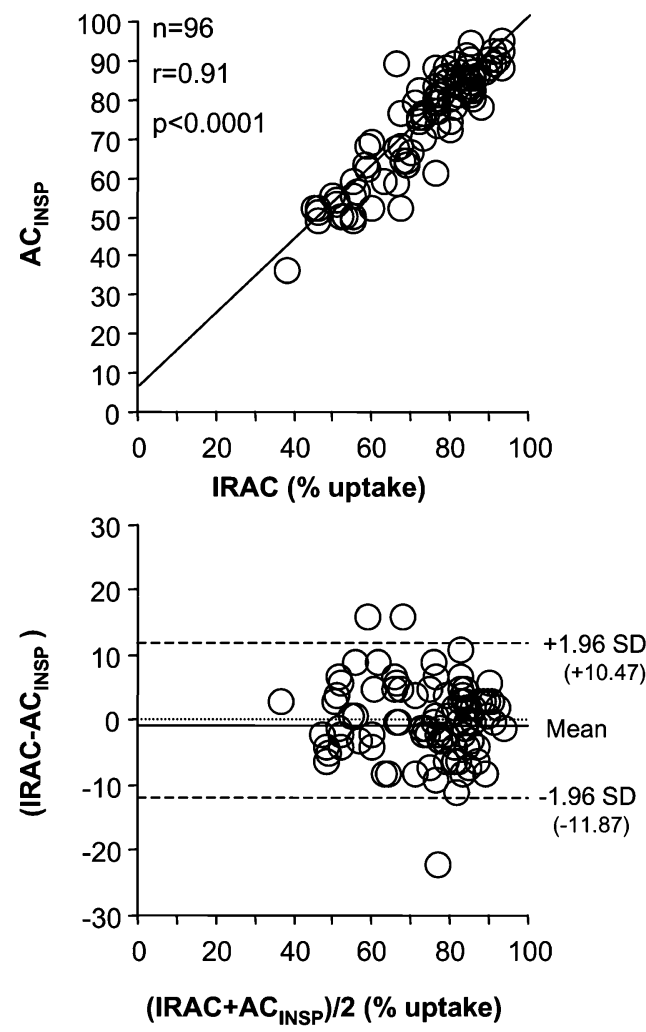

d

Lateral

IRAC vs AC INSP
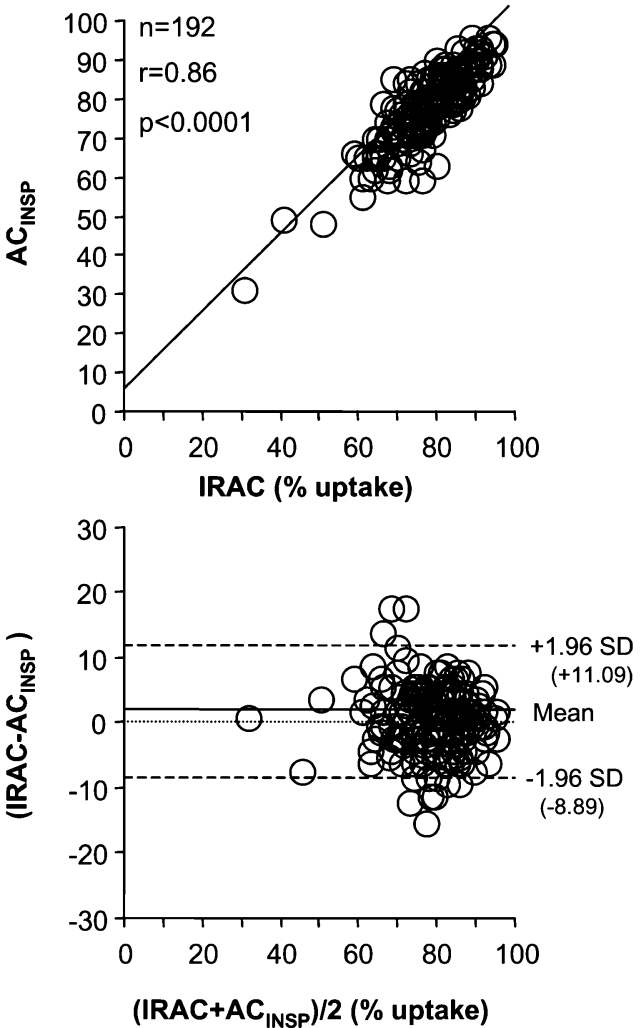

Septum

IRAC vs AC $_{\text {EXP }}$
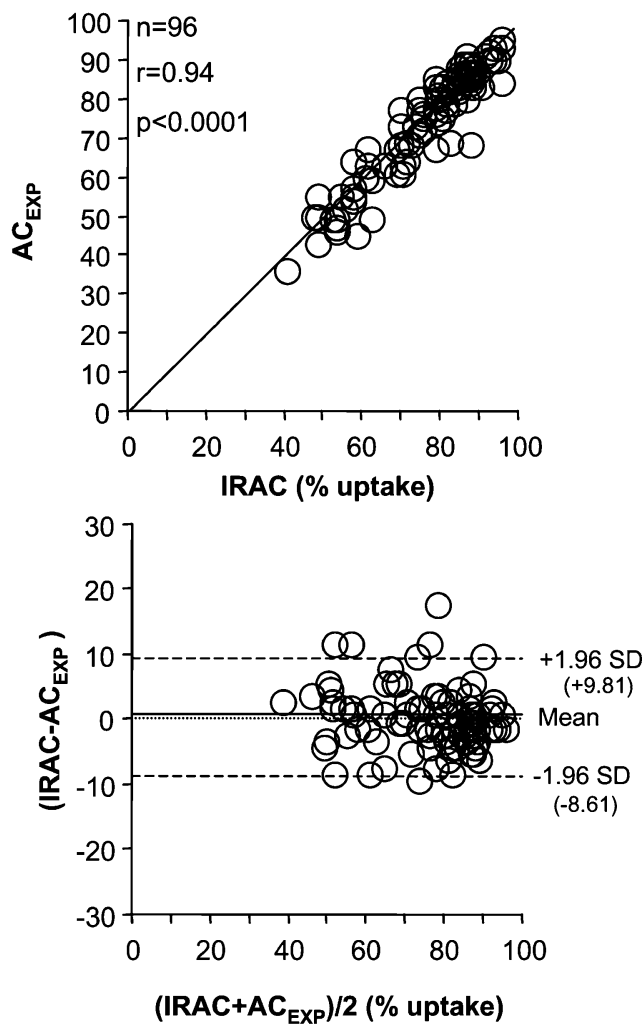

Lateral

IRAC vs AC $_{\text {EXP }}$
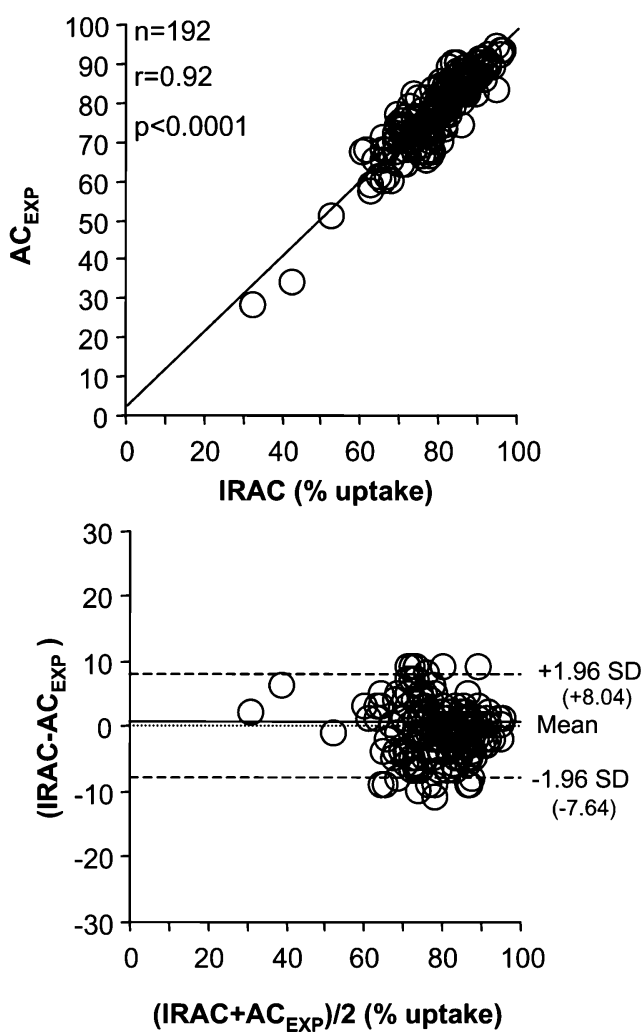
Fig. 2 (continued)
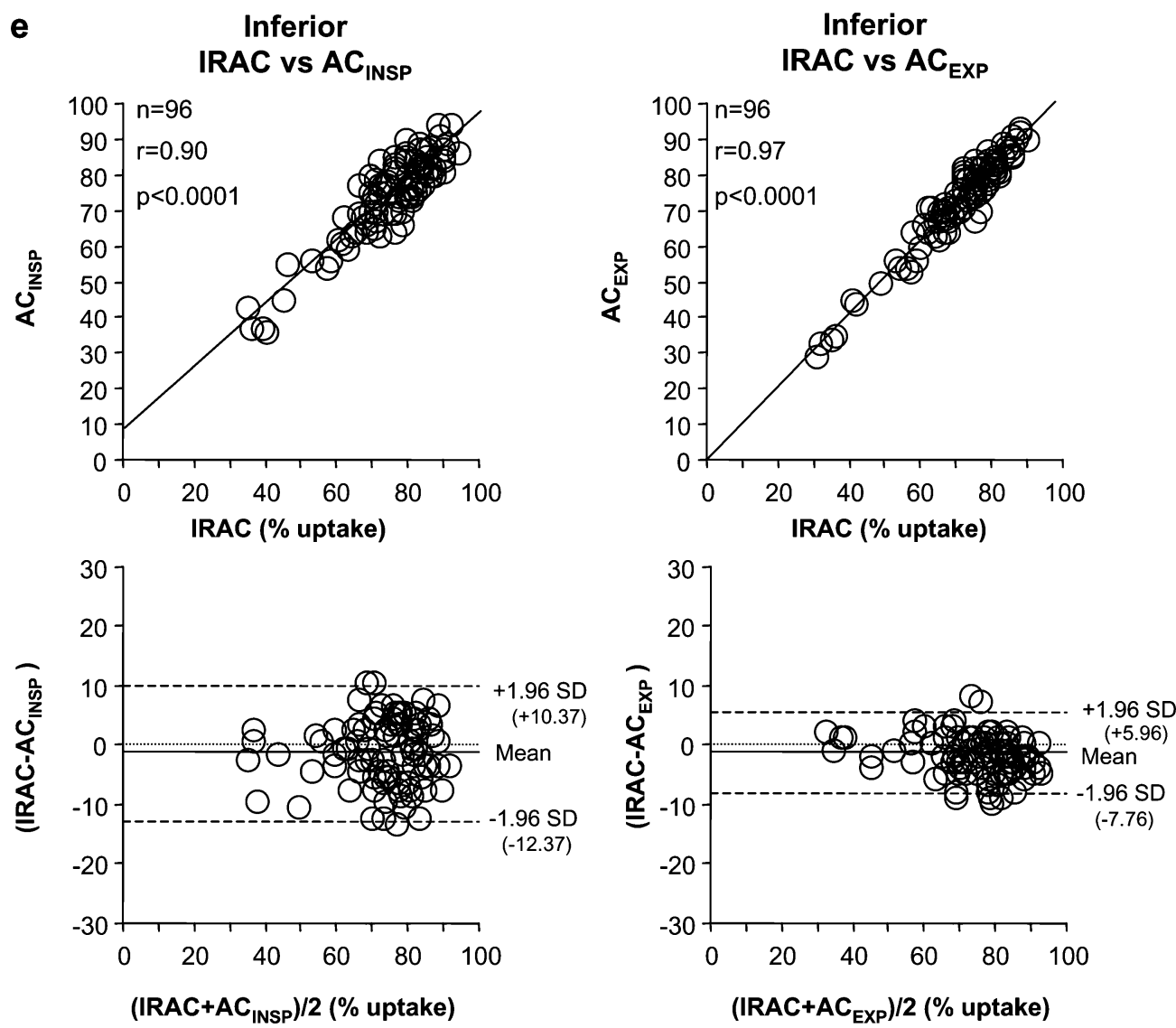

SDS was slightly inferior $(r=0.79, p<0.0001$ and $r=0.81$; $p<0.0001$, respectively). All perfusion abnormalities were supported by the corresponding findings on the $\mathrm{CT}$ angiogram (Table 2).

\section{Quantitative regional uptake values}

There was an excellent correlation between regional uptake values obtained by IRAC compared with $\mathrm{AC}_{\mathrm{INSP}}$ (apex, $r=0.92$; anterior, $r=0.85$; septal, $r=0.91$; lateral, $r=0.86$; inferior, $r=0.90$; all $p<0.0001$ ), with narrow limits of agreement (Fig. 2) and only a minimal (non-significant) overestimation of apical uptake values by $\mathrm{AC}_{\mathrm{INSP}}$. Correlation between IRAC and $\mathrm{AC}_{\mathrm{EXP}}$ was even higher (apex, $r=0.97$; anterior, $r=0.91$; septal, $r=0.94$; lateral, $r=0.92$; inferior, $r=0.97$; all $p<0.0001$ ) and limits of agreement closer compared with $\mathrm{AC}_{\mathrm{INSP}}$ (Fig. 2).

\section{Intra- and inter-observer reproducibility}

The high correlation between repeat measurements of regional uptake values obtained by $\mathrm{AC}_{\mathrm{INSP}}$ (apex, $r=0.95$; anterior, $r=0.80$; septal, $r=0.97$; lateral, $r=0.86$; inferior, $r=0.97$; all $p<0.0001$ ) and $\mathrm{AC}_{\mathrm{EXP}}$ (apex, $r=0.96$; anterior, $r=0.87$; septal, $r=0.94$; lateral, $r=0.80$; inferior, $r=0.98$; all $p<0.0001$ ) documented an excellent intra-observer reproducibility. Similarly, a high inter-observer agreement was obtained by $\mathrm{AC}_{\mathrm{INSP}}$ (apex, $r=0.80$; anterior, $r=0.80$; septal, $r=0.91$; lateral, $r=0.82$; inferior, $r=0.97$; all $p<0.0001$ ) and $\mathrm{AC}_{\mathrm{EXP}}$ (apex, $r=0.82$; anterior, $r=0.88$; septal, $r=0.90$; lateral, $r=0.78$; inferior, $r=0.95$; all $p<0.0001$ ).

\section{Coronary calcium score}

CCS data obtained during expiration from two patients were not amenable to interpretation owing to respiratory motion due to failure to follow the breath-hold commands. For the remaining 30 patients, no statistically significant difference was found for the mean Agatston score using CT data during inspiration (319 \pm 737 ; range, $0-2,555)$ and expiration (317 \pm 778 ; range, $0-2,940)$ (mean difference, $-12 \pm 93 ; p=\mathrm{NS})$.

\section{Discussion}

This study demonstrates for the first time that the use of CT data from CCS obtained with 64-slice CT allows accurate AC of myocardial SPECT MPI images. The attenuationcorrected SPECT images obtained using multislice CT data from CCS (either $\mathrm{AC}_{\mathrm{INSP}}$ or $\mathrm{AC}_{\mathrm{EXP}}$ ) resulted in identical clinical findings and conclusions compared with IRAC. This was underlined by the excellent correlation of the quantitative parameters between CCS data and IRAC. Furthermore, all perfusion defects were supported by the 
corresponding findings on the $\mathrm{CT}$ angiogram, confirming that no artefacts were introduced in our study population by either $\mathrm{AC}$ technique.

Comparison of regional perfusion uptake values with IRAC revealed a slightly superior agreement of $\mathrm{AC}_{\mathrm{EXP}}$ over $\mathrm{AC}_{\mathrm{INSP}}$, which was mainly evident for the apex, as this region is most prone to respiratory motion artefacts and partial volume effects [20]. This might be, at least in part, due to the fact that during acquisition of the low-dose CT, the upper abdominal organs as well as the heart and the lower mediastinum are scanned predominantly in a position that is close to that of expiration, as expiration constitutes the longest phase of the respiratory cycle [21]. As a consequence, this may produce inaccurate attenuation coefficients during maximum inspiration. Our results are in line with a recent study by Utsunomiya et al. [12], who found that the accuracy of AC was higher with use of a free-breathing and post-exhalation breath-hold protocol during CT scanning than with the inspiration breath-hold protocol. Similarly, Goerres et al. [11] reported that the normal postexhalation breath-hold protocol for CT acquisition proved superior compared with deep inspiration for PET-CT image registration. Nevertheless, differences between $\mathrm{AC}_{\mathrm{INSP}}$ and $\mathrm{AC}_{\mathrm{EXP}}$ were minimal in our study, as accurate correction for misalignment was performed for each individual scan and $\mathrm{AC}$ method.

Our results may have implications for future cardiac SPECT-CT protocols for several reasons. First, the integration of high-performance multislice $\mathrm{CT}$ into hybrid SPECT-CT devices will allow combined assessment of MPI and coronary anatomy or CCS. The latter-acquired within seconds as opposed to the minutes needed for the attenuation maps by low-resolution X-ray CT-improves the efficiency of the scan protocol and increases patient throughput. Second, our results suggest that the CCS should be acquired during expiration instead of inspiration, in order to enable its use as an attenuation correction map for MPI SPECT. In fact, $\mathrm{AC}_{\mathrm{EXP}}$ proved superior to $\mathrm{AC}_{\mathrm{INSP}}$ without affecting the accuracy of the Agatston score.

We acknowledge the following limitations of our study. First, we did not perform scatter correction although AC may amplify the scatter spillover into nearby myocardium, typically the inferior septal wall, from splanchnic activity [17]. However, visual and quantitative analysis revealed excellent agreement of the different $\mathrm{AC}$ methods and all findings were supported by the CT angiogram. Second, while $\mathrm{AC}_{\mathrm{EXP}}$ was found to be slightly superior to $\mathrm{AC}_{\mathrm{INSP}}$ with regard to quantitative comparison with IRAC, $\mathrm{AC}_{\mathrm{EXP}}$ was technically not successful in two of the 32 study participants as these two did not properly follow the breathhold command at expiration, while there was no problem with inspiration. Owing to the very low number of unsuccessful scans, it is not entirely clear whether breathhold commands at expiration for $\mathrm{AC}$ are more demanding while breath-hold commands at inspiration are generally followed very well. Third, regional quantitative uptake values were only analysed for stress scans as we expected the low-dose scan to be more susceptible to artefacts induced by different $\mathrm{AC}$ protocols. The fact that we found no differences between the various AC methods, although we confined this analysis to the low-dose scans, strengthens the finding of equivalence between $\mathrm{AC}$ from $\mathrm{CCS}$ compared with IRAC.

\section{Conclusion}

Attenuation maps from CCS allow accurate AC of SPECT MPI images. $\mathrm{AC}_{\mathrm{EXP}}$ proved superior to $\mathrm{AC}_{\mathrm{INSP}}$, suggesting that in hybrid scans CCS may be performed during normal expiration to allow its additional use for $\mathrm{AC}$ of cardiac SPECT MPI images.

Acknowledgements. Philipp A. Kaufmann was supported by a grant from the Swiss National Science Foundation (SNSF professorship grant No. PP00A-68835 and grant No. 31-68386). Hatem Alkadhi and Lotus Desbiolles were supported by the National Center of Competence in Research, Computer Aided and Image Guided Medical Interventions (NCCR CO-ME) of the Swiss National Science Foundation.

We are grateful to our head radiographer, Gabi Hasler, for her excellent technical assistance.

\section{References}

1. Fleischmann S, Koepfli P, Namdar M, Wyss CA, Jenni R, Kaufmann PA. Gated ${ }^{99 \mathrm{~m}}$ Tc-tetrofosmin SPECT for discriminating infarct from artifact in fixed myocardial perfusion defects. J Nucl Med 2004;45:754-9

2. Hendel RC, Corbett JR, Cullom SJ, DePuey EG, Garcia EV, Bateman TM. The value and practice of attenuation correction for myocardial perfusion SPECT imaging: a joint position statement from the American Society of Nuclear Cardiology and the Society of Nuclear Medicine. J Nucl Cardiol 2002;9:135-43

3. Bailey DL, Hutton BF, Walker PJ. Improved SPECT using simultaneous emission and transmission tomography. J Nucl Med 1987;28:844-51

4. Berman DS, Kiat H, Friedman JD, Wang FP, van Train K, Matzer L, et al. Separate acquisition rest thallium-201/stress technetium-99 m sestamibi dual-isotope myocardial perfusion single-photon emission computed tomography: a clinical validation study. J Am Coll Cardiol 1993;22:1455-64

5. Ficaro EP, Fessler JA, Shreve PD, Kritzman JN, Rose PA, Corbett JR. Simultaneous transmission/emission myocardial perfusion tomography. Diagnostic accuracy of attenuationcorrected ${ }^{99 \mathrm{~m}} \mathrm{Tc}$-sestamibi single-photon emission computed tomography. Circulation 1996;93:463-73

6. Bocher M, Balan A, Krausz Y, Shrem Y, Lonn A, Wilk M, et al. Gamma camera-mounted anatomical X-ray tomography: technology, system characteristics and first images. Eur J Nucl Med 2000;27:619-27

7. Masood Y, Liu YH, Depuey G, Taillefer R, Araujo LI, Allen S, et al. Clinical validation of SPECT attenuation correction using $\mathrm{x}$-ray computed tomography-derived attenuation maps: multicenter clinical trial with angiographic correlation. J Nucl Cardiol 2005;12:676-86

8. Fricke H, Fricke E, Weise R, Kammeier A, Lindner O, Burchert WA. A method to remove artifacts in attenuation-corrected myocardial perfusion SPECT introduced by misalignment between emission scan and CT-derived attenuation maps. J Nucl Med 2004;45:1619-25 
9. Namdar M, Hany TF, Koepfli P, Siegrist PT, Burger C, Wyss $\mathrm{CA}$, et al. Integrated PET/CT for the assessment of coronary artery disease: a feasibility study. J Nucl Med 2005;46:930-5

10. O'Connor MK, Kemp B, Anstett F, Christian P, Ficaro EP, Frey $\mathrm{E}$, et al. A multicenter evaluation of commercial attenuation compensation techniques in cardiac SPECT using phantom models. J Nucl Cardiol 2002;9:361-76

11. Goerres GW, Burger C, Kamel E, Seifert B, Kaim AH, Buck A, et al.Respiration-induced attenuation artifact at PET/CT: technical considerations. Radiology 2003;226:906-10

12. Utsunomiya D, Nakaura T, Honda T, Shiraishi S, Tomiguchi S, Kawanaka K, et al. Object-specific attenuation correction at SPECT/CT in thorax: optimization of respiratory protocol for image registration. Radiology 2005;237:662-9

13. Germano G, Kiat H, Kavanagh PB, Moriel M, Mazzanti M, Su HT, et al. Automatic quantification of ejection fraction from gated myocardial perfusion SPECT. J Nucl Med 1995;36: 2138-47

14. Cerqueira MD, Weissman NJ, Dilsizian V, Jacobs AK, Kaul S, Laskey WK, et al. Standardized myocardial segmentation and nomenclature for tomographic imaging of the heart: a statement for healthcare professionals from the Cardiac Imaging Committee of the Council on Clinical Cardiology of the American Heart Association. Circulation 2002;105:539-42
15. Sharir T, Germano G, Waechter PB, Kavanagh PB, Areeda JS, Gerlach J, et al. A new algorithm for the quantitation of myocardial perfusion SPECT. II: Validation and diagnostic yield. J Nucl Med 2000;41:720-7

16. Germano G, Kavanagh PB, Waechter P, Areeda J, Van Kriekinge S, Sharir T, et al. A new algorithm for the quantitation of myocardial perfusion SPECT. I: Technical principles and reproducibility. J Nucl Med 2000;41:712-9

17. Ficaro EP, Fessler JA, Ackermann RJ, Rogers WL, Corbett JR, Schwaiger M. Simultaneous transmission-emission thallium201 cardiac SPECT: effect of attenuation correction on myocardial tracer distribution. J Nucl Med 1995;36:921-31

18. Agatston AS, Janowitz WR, Hildner FJ, Zusmer NR, Viamonte M Jr, Detrano R. Quantification of coronary artery calcium using ultrafast computed tomography. J Am Coll Cardiol 1990; $15: 827-32$

19. Bland JM, Altman DG. Statistical methods for assessing agreement between two methods of clinical measurement. Lancet 1986;1:307-10

20. Fricke E, Fricke H, Weise R, Kammeier A, Hagedorn R, Lotz $\mathrm{N}$, et al. Attenuation correction of myocardial SPECT perfusion images with low-dose CT: evaluation of the method by comparison with perfusion PET. J Nucl Med 2005;46:736-44.

21. Wade OL. Movements of the thoracic cage and diaphragm in respiration. J Physiol 1954;124:193-212 